\title{
Use of Steroids by SUT Students
}

\author{
K. Vrenjo \\ Lecturer; Faculty of Movement Sciences, Sports University of Tirana \\ 0. Petri \\ Lecturer; Faculty of Rehabilitation Sciences, Sports University of Tirana
}

\begin{abstract}
Through the survey carried out with SUT students we seek to assess how much they know about steroids, their use, benefits and side effects. These students are active: as athletes in various disciplines, in coaching and after graduation as teachers of physical education and health. 201 SUT students, 113 males and 88 females were randomly included in the survey. This topic was selected because these substances can be easily found and used without prescription of a specialist in Albania. Raising the awareness of SUT students is important for their health and as future educators serve as a promoter of healthy living to every child at school or peoples engaged in sports.
\end{abstract}

Keywords: steroids, students, SUT

\section{Introduction}

Anabolic androgenic steroids AAS are synthetic derivatives of testosterone, which is the primary male sex hormone. Anabolic androgenic steroids are used to enhance athletic performance and appearance. Adverse effects include those on the liver, serum lipids, psyche/behaviour and reproductive system.

Through the survey of the target group, students of SUT, we seek to assess how much information they have about steroids, their use, benefits and side effects. Students are athletes of various disciplines, are engaged in physical activity as part of a job, stay active for personal pleasure and the rest engage in physical activity only during the school practice classes. After graduation they are employed as teachers of physical education and health, as personal trainers and as coaches in various sports disciplines serving as a link between physical activity and health of any age.

Public health problems are also increasing in our country, given the IPH's National Health Report 2014. Meanwhile, according to studies carried out it has been confirmed that physical activity and exercise improves quality of life, prevents and is used to treat many diseases but mainly in the field of mortality reduction, CVD 
risk factors and micro vascular complications, etc. For all these reasons, there is an increasing interest in our country to include the population in physical activity, therefore our mission is to prepare professionals with knowledge and information on how to select accordingly physical exercise, its duration by taking into consideration certain health conditions, nutrition and supplements, etc, and thus ensuring satisfactory quality and results for each individual.

\section{Material and methods}

In the survey there were included 201 SUT students from all years of study, aged 19-26, who were randomly selected and the respondents are 113 or 56.2 males and 88 or $43.8 \%$ females.

This topic was selected because: according to our knowledge there is no national study on the use of steroids for the Albanian population, we addressed a contingent with a young age and that engage in physical activity as these two factors have a considerable affect in the use of steroids. Moreover, in the school curriculum these substances are dealt with only in a few lectures in the third year of bachelor studies. Another reason is that these substances in our country can be easily found in the black market or authorized stores and are used without the recommendation of a specialist doctor as the latter is nonexistent.

The questionnaire used was structured and drafted in collaboration with psychologists and with the aim to be applied to students who deal with public health (e.g. nurses) and our intention is to understand whether we should intervene in the curricula or if the information they actually receive is sufficient.

The survey was distributed, filled and collected via Google forms.

\section{Results and discussions}

We need to clarify that the period when the questionnaire was distributed was during the pandemic, thus students have less opportunity to perform physical activity or to be briefed before answering the questionnaire as this survey was online.

According to the responses given we have obtained the following important results:

According to the place of residence 183 (91.04\%) live in cites and 18 (8.96\%) live in villages, so they have the opportunity to engage in physical activities.

This is confirmed in the next question where 177 (88.1\%) engage in physical activity while $24(11.9 \%)$ don't. Those who engage in physical activity at a frequency of 0 2 times a week do exercise / physical activity 55 (27.4\%) of them and 66 (32.8\%) do fitness, so they are not athletes but amateurs and the rest 80 respondents $(39.8 \%)$ are engaged in various disciplines.

Q: Do you know the substances called "steroids"? A: Yes = 183(91.0\%), No = 18(9.0\%) 
Q: If yes, where did you get the information from?

A: Internet $=140$ (69.9\%.): TV $=35:$ Friends $=73:$ Literature $=45:$ Medical staff $=$ 26:(12.9\%.)

Based on the answers received we assume that the students may have received and looked up for information on steroids at the time of completing the questionnaire. This raises the need for curriculum review not only at the University but perhaps even in the earlier stages.

Q: Do you think anabolic steroids increase muscle mass?

A: Yes = 170 (84.6\%), No = 31 (15.4\%),

This is a topic of conversation between people who engage in physical activity, strengthening the fact that $(36.3 \%)$ have received information from friends and colleagues.

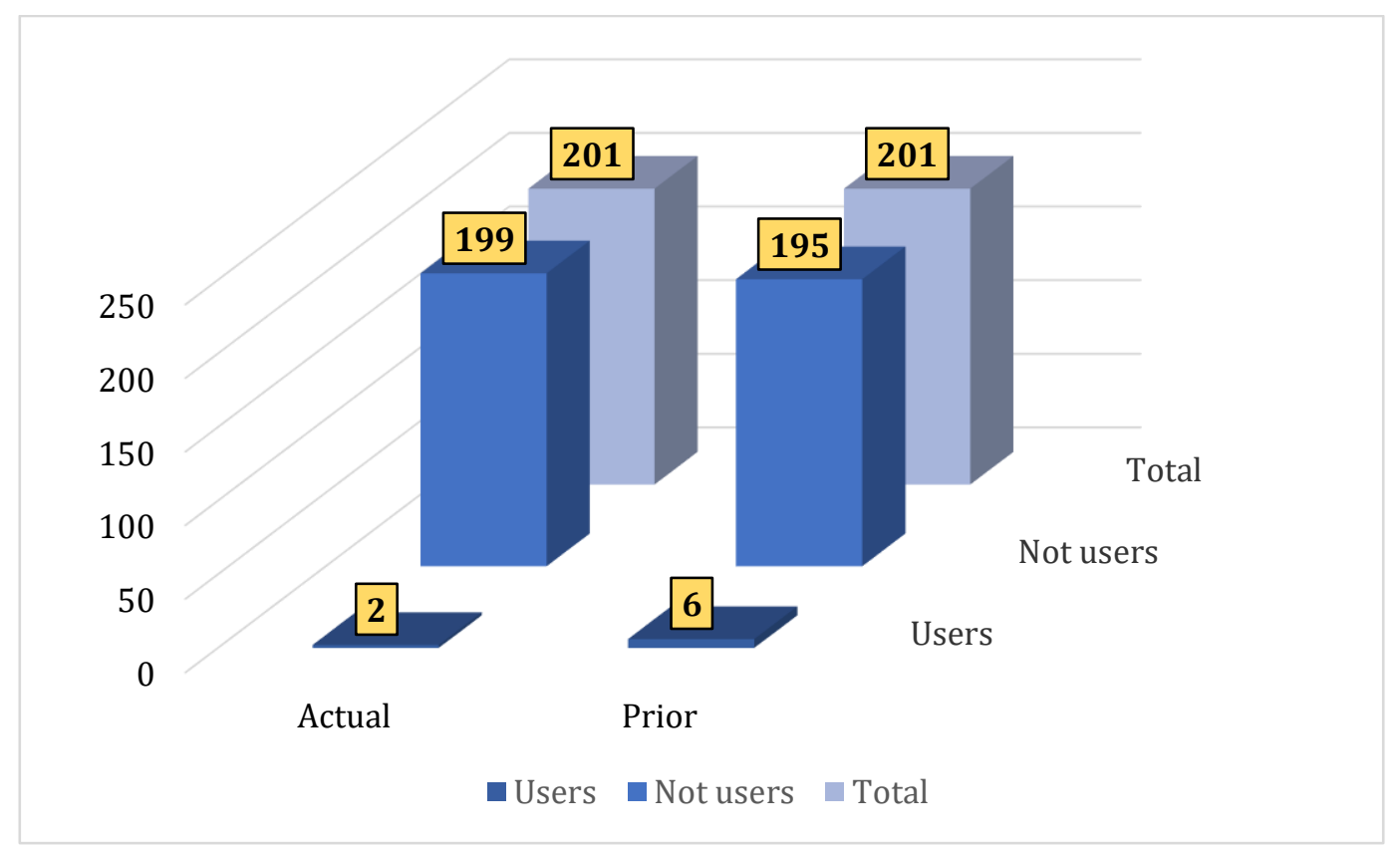

Diagram 1: Previous and Actual users of AAS 


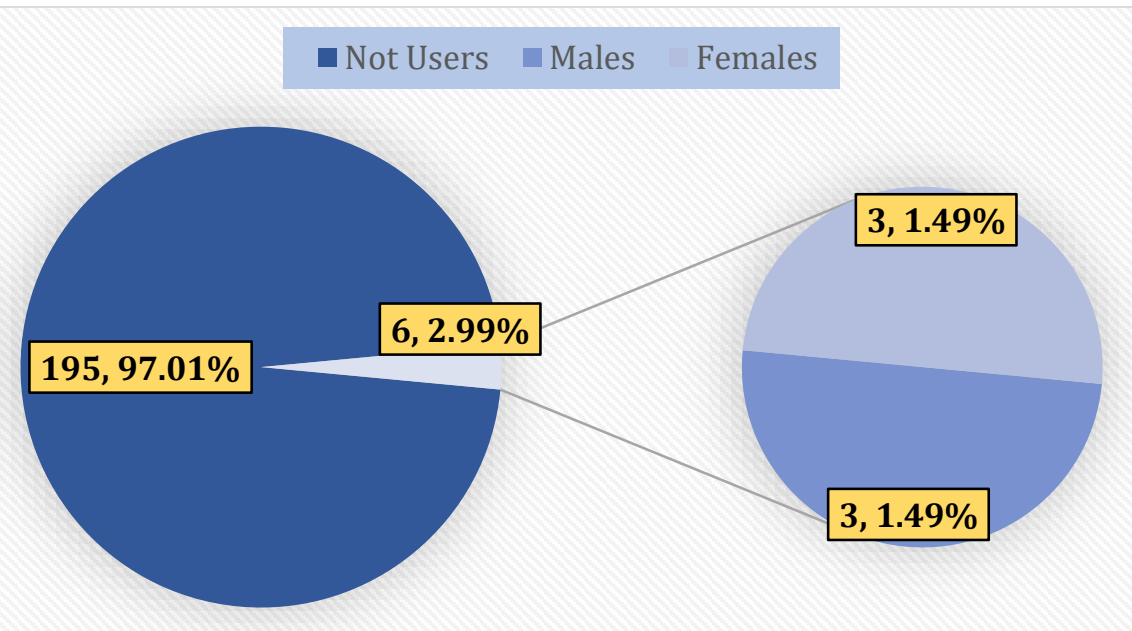

Diagram 2: AAS Users divided between males and females

Diagrams 1,2 show that the users of AAS are actually only $2(1.0 \%)$ but 4 other respondents have consumed them sometimes in their lives but fortunately are at a low percentage respectively at $2.99 \%$. However, it needs to be highlighted that both genders have consumed them equally. Another fact worth highlighting is that these 6 subjects are engaged in a sports discipline.

Q: When did you first use AAS?

6 answers are given by respondents aged between 15-20 years which correspond to other studies which state that this is the age group with a higher tendency to use these substances.

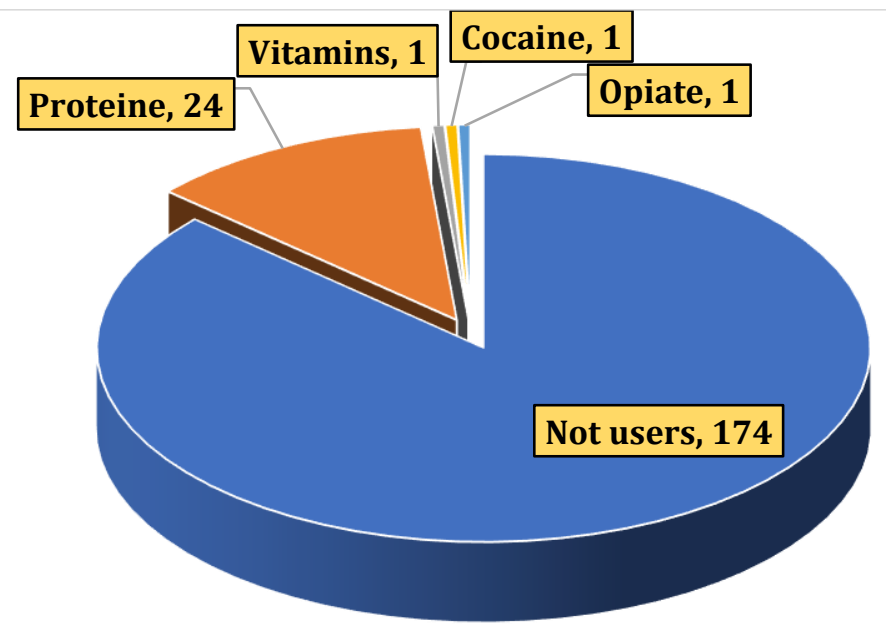

- Not users $\square$ Proteine $\square$ Vitamins $\amalg$ Cocaine $\square$ Opiate 
Diagram 3 Users of others substances

As shown in above diagram the question: Do you use substances other than anabolic? Is answered accordingly: Yes $=27(23=$ Protein as supplement: $1=$ anabolic hormone + Protein as supplements: 1 = Vitamins: 1 = Cocaine: 1 = Opiate)

No $=174(86.6 \%)$,

The answers to this question emphasize once again that our students tend to use supplements, mostly healthy, but still it is worrying the fact that these are taken without a doctor's recommendation. $12 \%$ of them use supplements in the form of supplements, of which $50 \%$ go to the gym, so they tend to increase their use when exercising at the gym, as well as the ones who engage in football and boxing.

Q: Do you know anyone who uses AAS? A: Yes = 66 (32.8\%), No =135 (67.2\%),

Q: What is the main way in which you / your acquaintances obtain these substances?

A: Shopping in authorized stores $=104(60.5 \%)$, at illegal market $=68(39.5 \%)$, No answer $=29$

Q: How are these substances taken?

Orally=70: Injection = 106: No answer $=25$

Q: Do you know the side effects of AAS on physical health?

A: Yes $=20$ (9.9\%), No = 65 (32.4\%), No answer $=116(57.7 \%)$,

$57.7 \%$ have not answered indicating that they do not know the side effects as they do not even know these substances. 


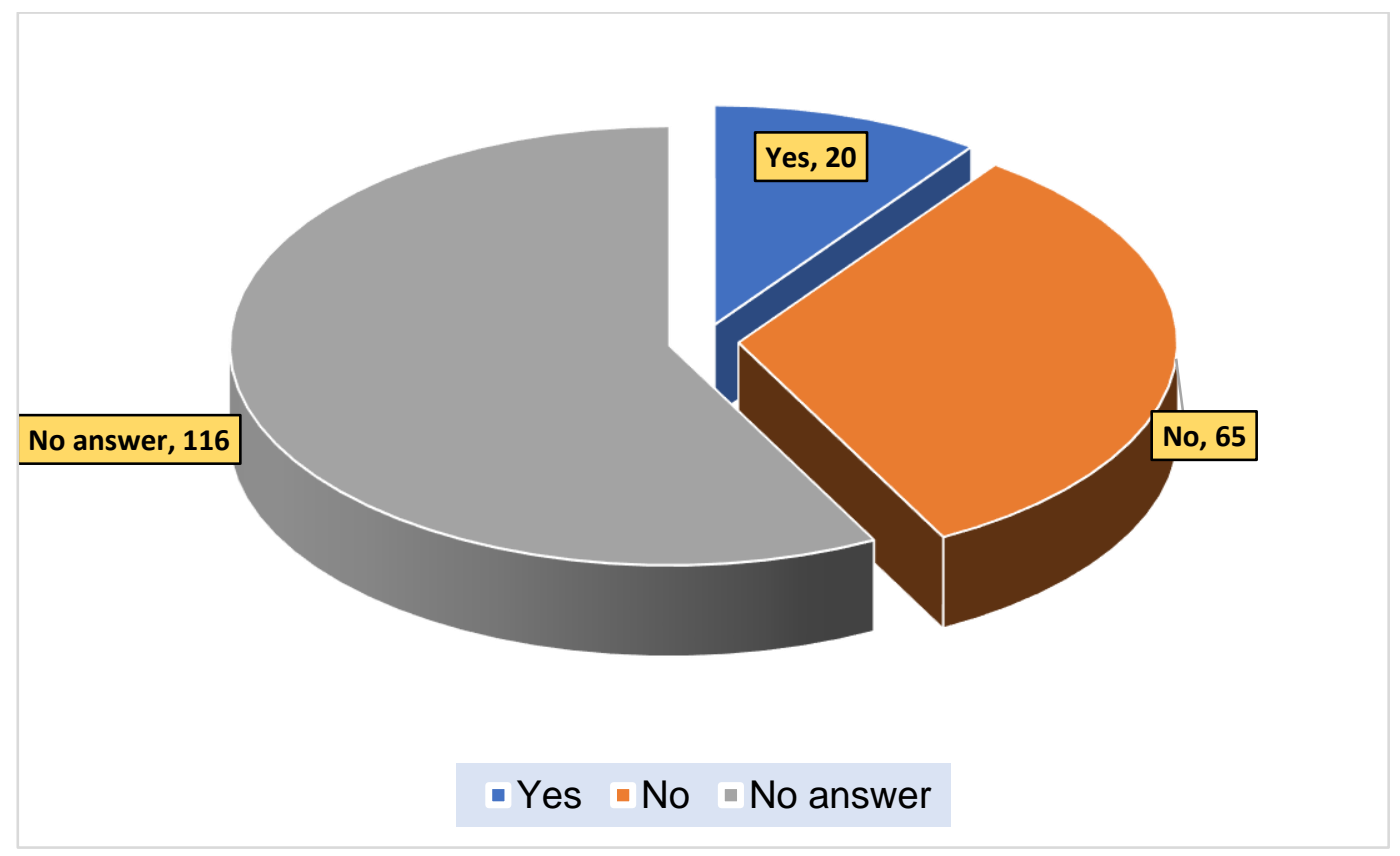

Diagram 4: Information about side effects on physical health

This fact is clarified by the answers to the following two questions:

- What is the reason that these substances are used, according to you?

A:

To improve physical appearance $=86$

To improve physical performance $=105$

No answer $=10$

Q: Do you think anabolic affect in the following? (choose one or several alternatives according to your information)

A:

Body weight $=80$

Muscle size $=138$

Muscle strength $=81$

Increases fertility $=24$

Increases breast $=39$

Affect the heart $=98$

Cause acne $=32$ 
Cause behaviour change $=45$

Cause mood swings $=48$

Cause different types of cancer $=48$

\section{Results}

The results of questionnaire are as follows:

- 177 engage in physical activity, 24 don't.

- Q: Do you know the substances called "steroids"? A: Yes = 183, No = 18

- Q: Do you use these substances? A: Yes $=2$, No $=199$

- Q: Do you use substances other than anabolic? A: Yes $=13$, No $=188$

- Q: Do you know anyone who uses AAS? A: Yes = 66, No =135

- Q: How are these substances taken?

- A: Orally=70: Injection =106: No answer $=25$

- Q: What is the reason that these substances are used, according to you?

A:

To improve physical appearance $=86$

To improve physical performance $=105$

No answer $=10$

\section{Conclusion}

Students have information but not thorough knowledge on what steroids are, what they cause in the body; they know very little about the positive effects and almost all the negative ones, so we suggest that the curriculum should be reviewed not only at University level but maybe even earlier.

The student subjects who practice a certain discipline are $39.8 \%$, so on the light of this fact the users of steroids are in small percentage only $2.99 \%$.

Our students tend to use supplements, mostly healthy, but again worrying is the fact that they are taken without a doctor's recommendation.

As we suspected in our country steroid substances are also found on the black market as $39.5 \%$ of the respondents have confirmed it.

This study exceeded our expectations by raising the claim that perhaps the time has come to undertake a national study on the use of steroids for people who engage in physical activity, adolescents or the Albanian population in general as abuse of these substances is considered a matter of public health. At the same time, in order to prevent the use of AAS, we must insist on improving the knowledge and awareness among health care workers and employees engaged in physical activity, educational interventions, by updating respective legislation and using appropriate doping screening tests. 


\section{Disclosure Statement}

The author declared no potential conflicts of interest concerning the research, authorship, and/or publication of this article

\section{References}

[1] IPH 2014. National Health Report (Health status of the Albanian people), Risk Factors, High body mass index, Hypertension, High blood glucose level, pp.111 - 135

[2] INSTAT 2010. Demographic and Health Survey in Albania 2008 - 2009. IPH, Adult Health, Hypertension, Prevalence of hypertension, pp. $231-236$

[3] Michael S Bahrke 1, Charles E Yesalis, 2004, Abuse of anabolic androgenic steroids and related substances in sport and exercise doi: 10.1016/j.coph. 05.006. 2004 Dec;4(6):614-20.

[4] Giuseppe Davide Albano 1, Francesco Amico 1, Giuseppe Cocimano 1, Aldo Liberto 1 , Francesca Maglietta 2 , Massimiliano Esposito 1, Giuseppe Li Rosi 3 , Nunzio Di Nunno 4, Monica Salerno 1, Angelo Montana 1, 2021 Adverse Effects of Anabolic-Androgenic Steroids: A Literature Review . doi: 10.3390/healthcare9010097. 2021 Jan 19;9(1):97. PMID: 33477800 . PMCID: PMC7832337

[5] Giuseppe Bertozzi 1 , Monica Salerno 2 , Cristoforo Pomara 2 , Francesco Sessa 3 , 2019, Neuropsychiatric and Behavioral Involvement in AAS Abusers. A Literature Review : A Literature Review Medicina (Kaunas) 2019 Jul 22;55(7):396. doi: 10.3390/medicina55070396. PMID: 31336641 PMCID: PMC6681542 DOI: 10.3390/medicina55070396

[6] Forbes, G.B., 1985. The effect of anabolic steroids on lean body mass: the dose response curve. Metabolism 34, 571-573.

[7] Bahrke, M.S., Yesalis, C.E., Kopstein, A.N., Stephens, J.A., 2000. Risk factors associated with anabolic-androgenic steroid use among adolescents. Sports Med. 29, 397-405

[8] Király, C.L., 1987. Effect of testosterone and anabolic steroids onthe size of sebaceous glands in power athletes. Am. J. Dermatopathol. 9, 515-519

[9] Wilson, J.D., 1988. Androgen abuse by athletes. Endocr. Rev. 9, 181-199

[10] Eklof, A.C., 2003. The anti-doping hot-line, a means to capture the abuse of doping agents in the Swedish society and a new service function in clinical pharmacology. Eur. J. Clin. Pharmacol. 59, 571-577. 